\title{
Bacteroides fragilis metabolises exopolysaccharides produced by bifidobacteria
}

\author{
David Rios-Covian ${ }^{1 *}$, Isabel Cuesta ${ }^{2}$, Jorge R. Alvarez-Buylla², Patricia Ruas-Madiedo ${ }^{1}$, Miguel Gueimonde
} and Clara G. de los Reyes-Gavilán

\begin{abstract}
Background: Bacteroides fragilis is the most frequent species at the human intestinal mucosal surface, it contributes to the maturation of the immune system although is also considered as an opportunistic pathogen. Some Bifidobacterium strains produce exopolysaccharides (EPS), complex carbohydrate polymers that promote changes in the metabolism of $B$. fragilis when this microorganism grows in their presence. To demonstrate that $B$. fragilis can use EPS from bifidobacteria as fermentable substrates, purified EPS fractions from two strains, Bifidobacterium longum E44 and Bifidobacterium animalis subsp. lactis R1, were added as the sole carbon source in cultures of B. fragilis DSMZ 2151 in a minimal medium. Bacterial counts were determined during incubation and the evolution of organic acids, short chain fatty acids (SCFA) and evolution of EPS fractions was analysed by chromatography.
\end{abstract}

Results: Growth of B. fragilis at early stages of incubation was slower in EPS than with glucose, microbial levels remaining higher in EPS at prolonged incubation times. A shift in metabolite production by B. fragilis occurred from early to late stages of growth, leading to the increase in the production of propionate and acetate whereas decrease lactate formation. The amount of the two peaks with different molar mass of the EPS E44 clearly decreased along incubation whereas a consumption of the polymer R1 was not so evident.

Conclusions: This report demonstrates that $B$. fragilis can consume some EPS from bifidobacteria, with a concomitant release of SCFA and organic acids, suggesting a role for these biopolymers in bacteria-bacteria cross-talk within the intestine.

Keywords: Bacteroides fragilis, Exopolysaccharides, Bifidobacterium, MALLS, Heteropolysaccharides

\section{Background}

The colonic microbiota is a complex community whose metabolic activity influences our health and nutritional status through diverse pathways [1]. Microbiota of adult healthy people is dominated by the phyla Firmicutes and Bacteroidetes, even though the composition at the species level is highly variable among individuals $[2,3]$. Members of this microbiota are able to degrade complex polysaccharides and, therefore, genes involved in the degradation and consumption of these compounds are

\footnotetext{
* Correspondence: drioscovian@ipla.csic.es

'Probiotics and Prebiotics Group, Department of Microbiology and

Biochemistry of Dairy Products, Instituto de Productos Lácteos de Asturias,

Consejo Superior de Investigaciones Científicas (IPLA-CSIC), Villaviciosa,

Asturias, Spain

Full list of author information is available at the end of the article
}

widespread among the genomes of microbiota-resident species [4, 5]. In this respect, the genus Bacteroides has the ability to use a wide range of carbohydrates, this ability varying as depending on the species considered [6]. Bacteroides is an anaerobic, bile-resistant, non-spore forming, and Gram negative rod [7] that accounts for up to $20-50 \%$ of the total microbiota in most individuals [8]. Bacteroides fragilis is the most frequent species at the mucosal surface [9] and can contribute to the development and maturation of the host immune system [10]. This species has an extraordinarily good adaptability to environmental changes due to its capacity to regulate the cell surface [11]. Consequently B. fragilis is the clinical 
isolate most frequently found in systemic infections, this microorganism being then considered as an opportunistic pathogen [7].

Exopolysaccharides (EPS) are complex carbohydrates located outside the cell; some Bifidobacterium strains, as well as many other microorganisms, are able to produce these polymers [12]. The synthesis of these compounds in the intestine has not been demonstrated yet. However, it has been proven in vitro that the presence of bile, which is released to the small intestine during digestion, stimulates the production of EPS by bifidobacteria [13]. EPS could be constituted either by a unique type of monomer, named as homopolysaccharides (HoPS), or by more than one monosaccharide type, then known as heteropolysaccharides (HePS). All bifidobacterial EPS characterized until present are HePS [14].

Differential growth of members from the genus Bacteroides in the presence of EPS isolated from Lactobacillus and Bifidobacterium strains has been previously reported $[15,16]$. Particularly, two EPS fractions isolated from Bifidobacterium longum E44 and Bifidobacterium animalis subsp. lactis R1, have shown the capacity to act as fermentable substrates by the intestinal microbiota, thus promoting the increase of Bacteroides populations in faecal cultures $[15,17]$. Specific changes have been demonstrated as well in the metabolism of $B$. fragilis when grown in an undefined and complex medium in the presence of EPS E44 and R1 as compared with cultures in glucose [18]. Although these studies suggest that Bacteroides can use bifidobacterial EPS as fermentable substrates, this had not yet been effectively proven. In the present work, we assessed this question by testing the ability of $B$. fragilis DSMZ 2151 to growth in a minimal medium at the expenses of EPS from bifidobacteria when they are the sole carbon and energy source available.

\section{Methods}

\section{Bacterial strains and culture media}

Frozen stocks of $B$. fragilis DSMZ 2151 (DSMZ bacterial pure collection, Braunschewig, Germany) were reactivated in Gifu anaerobic medium (GAM) broth (Nissui Pharmaceutical Co., Tokyo, Japan) supplemented with $0.25 \%$ (w/v) L-cysteine (Sigma Chemical co., St. Louis, MO, USA) (named GAMc) and incubated overnight at $37{ }^{\circ} \mathrm{C}$ in an anaerobic cabinet (Mac 100; Don Whitley Scientific, West Yorkshire, UK) under a $10 \% \mathrm{H}_{2}, 10 \%$ $\mathrm{CO}_{2}$, and $80 \% \mathrm{~N}_{2}$ atmosphere. $\mathrm{pH}$ free batch cultures of $B$. fragilis were performed in a modified minimal medium (MM) previously used in B. fragilis metabolism studies [19]. Briefly, the medium contained per litre: $\left(\mathrm{NH}_{4}\right)_{2} \mathrm{SO}_{4}$, 1 g; $\mathrm{KH}_{2} \mathrm{PO}_{4}, 0.9 \mathrm{~g} ; \mathrm{NaCl}, 0.9 \mathrm{~g} ; \mathrm{CaCl}_{2} \cdot 2 \mathrm{H}_{2} 0,26.5 \mathrm{mg}$; $\mathrm{MgCl} \cdot 6 \mathrm{H}_{2} \mathrm{O}, 20 \mathrm{mg} ; \mathrm{MnCl}_{2} \cdot 4 \mathrm{H}_{2} \mathrm{O}, 10 \mathrm{mg} ; \mathrm{FeSO}_{4} \cdot 7 \mathrm{H}_{2} \mathrm{O}$, $4 \mathrm{mg}$; $\mathrm{CoCl}_{2} \cdot 6 \mathrm{H}_{2} \mathrm{O}, 1 \mathrm{mg}$; resazurin, $1 \mathrm{mg}$; vitamin $\mathrm{B}_{12}$,
$10 \mu \mathrm{g}$; vitamin $\mathrm{K}_{1}, 2 \mathrm{mg}$; and haemin, $4 \mathrm{mg}$. MM was supplemented with $0.5 \%(\mathrm{w} / \mathrm{v})$ of glucose, EPS E44 or EPS R1 and the final $\mathrm{pH}$ of the medium ranged between 7.6 and 7.9. MM was inoculated with a $1 \%(\mathrm{v} / \mathrm{v})$ overnight culture of B. fragilis in GAMc in a final volume of $10 \mathrm{~mL}$. A culture of $B$. fragilis inoculated in MM without carbon source added was used as a negative control. Potential changes over time in the characteristics of the EPS fractions during incubation not due to the microbial action were monitored in uninoculated MM added with EPS. Bacterial growth was monitored by counting in agar-GAMc plates at $0,24,48,72$ and $144 \mathrm{~h}$ of incubation. Experiments were run in triplicate using the same inoculum in all conditions.

\section{EPS isolation}

The EPS fractions were obtained from the strains $B$. animalis subsp. lactis IPLA R1 [20], a dairy origin strain, and B. longum IPLA E44 [21], a faecal isolate from a healthy adult faeces. Cellular biomass was harvested with ultrapure water from agar-MRS (Biokar, Allone, France) plates with $0.25 \%(w / v)$ L-cysteine (Sigma) and incubated for 3 days at $37{ }^{\circ} \mathrm{C}$ under anaerobic conditions [22]. In brief, EPS was separated from the cellular biomass by gently stirring overnight with one volume of $2 \mathrm{M} \mathrm{NaOH}$ at room temperature. Then, cells were removed by centrifugation and EPS were precipitated from the supernatant with two volumes of absolute cold ethanol for $48 \mathrm{~h}$ at $4 \mathrm{C}$. After centrifugation at 10,000 $\mathrm{x} g$ for $30 \mathrm{~min}$ at $4 \mathrm{C}$, the EPS fraction was resuspended in ultrapure water and dialyzed against water during 3 days in 12 - to $14-\mathrm{kDa}$ molecular weight cut off dialysis tubes (Sigma). The protein content of the polymers was determined by the BCA protein assay kit (Pierce, Rockford, IL, USA) following the manufacturer's instructions. Finally, EPS fractions were freeze-dried. The EPS E44 and R1 fractions contained $2.25 \%$ and $1.99 \%$ protein, respectively.

To check the purity of the EPS and test for the eventual presence of bacterial glycogen, EPS E44 and R1 fractions and glycogen (Roche, Switzerland) were digested with $\alpha$ amylase (Sigma, USA) and/or pullulanase (Sigma, USA) and degradation profiles were compared. Digested samples were run in a TLC gel as described by Koropatkin and Smith [23]. In brief, each reaction mixture contained $1 \mathrm{mg} / \mathrm{mL}$ of substrate (EPS R1, EPS E44 or commercial glycogen) and $22 \mu \mathrm{g} / \mathrm{mL}$ of one or both enzymes in a $20 \mathrm{mM}$ HEPES buffer. After $4 \mathrm{~h}$ of digestion at $37^{\circ} \mathrm{C}, 6 \mu \mathrm{l}$ of each sample reaction were placed and dried in a TLC Silica Gel 60 (Merk, Germany). Gels were transferred to a solvent chamber with a 3:1:1 mixture of isopropanol: ethylacetate: water, run for $3 \mathrm{~h}$ and revealed by irrigating a $5 \%$ sulfuric dilution in ethanol and were dried at $120 \mathrm{C}^{\circ}$ for $10 \mathrm{~min}$. (Additional file 1: Figure S1). 


\section{Metabolite analysis and EPS molar mass distribution and quantification}

Organic acids (lactic, succinic and formic) formed during incubation were analysed by HPLC. Cell-free supernatants from cultures were filtered $(0.2 \mu \mathrm{m})$ and quantified using an Alliance 2695 module injector, a PDA 966 photodiode array detector, a 2414 differential refractometer detector and the Empower software (Walters, Mildford, MA), following the chromatographic conditions described previously [22]. The weight average molar mass $\left(\mathrm{M}_{\mathrm{w}}\right)$ distribution of EPS fractions was determined in the same equipment by size-exclusion chromatography (SEC) using two different columns placed in series, TSK-Gel G3000 PW $\mathrm{xL}_{\mathrm{xL}}$ and TSK-Gel G5000 PW $\mathrm{PL}_{\mathrm{xL}}$ (Supelco-Sigma) and the multiangle laser light scattering (MALLS) detector DawnHeleos II (Wyatt Europe GmbM, Dembach, Germany) as described previously [22]. The EPS peaks were detected and quantified with the refractive index detector, using standards of dextran (Fluka-Sigma) of different molar masses; the presence of proteins was monitored through a PDA detector set at $220 \mathrm{~nm}$ [17]. Short chain fatty acids (SCFA; acetic and propionic) were quantified in the supernatants by Gas Chromatography (GC) using a system composed of a $6890 \mathrm{~N}$ gas chromatograph (Agilent Technologies Inc., Palo Alto, CA, USA) connected with a FID detector (Agilent) and a mass spectrometry (MS) $5973 \mathrm{~N}$ detector (Agilent) as described previously [15]. Concentrations were expressed in millimolar (mM).

\section{Statistical analysis}

One way ANOVA statistical tests was run to compare the evolution of the different parameters analysed along time or among cultures with the different carbon and energy sources by means of the IBM SPSS software, version 22.00 (IBM, Armonk, New York, USA). SNK post-hoc test was used when required.

\section{Results}

The digestion pattern of EPS fractions E44 and R1 with the enzymes $\alpha$-amylase (endohydrolysis of 1,4- $\alpha$-Dglucosidic linkages) and pullulanase (endohydrolysis of 1,6- $\alpha$-D-glucosidic linkages), were clearly different from that of glycogen (Additional file 1: Figure S1). This indicates that the release of intracellular glycogen during the process of obtaining the EPS fractions is negligible. Therefore, the results obtained through our work should be related with EPS polymers.

The metabolite production and growth pattern of $B$. fragilis DSMZ 2151 was dependent on the carbon source present in the culture medium. The final $\mathrm{pH}$ attained with glucose $(6.52 \pm 0.16)$ was significantly lower than with EPS E44, EPS R1, and without carbon source $(p<0.05)$ $(7.62 \pm 0.02,7.66 \pm 0.01$ and $7.73 \pm 0.05$ respectively). The
$\mathrm{pH}$ values remained unchanged in $\mathrm{MM}$ without carbon source added whereas bacterial counts increased $1.3 \mathrm{log}$ units from time $0\left(7.02 \pm 0.08 \log \mathrm{ufc} \mathrm{mL}^{-1}\right)$ to $24 \mathrm{~h}$ of incubation in such conditions, probably due to the metabolic inertia of the inoculum (Table 1). At $24 \mathrm{~h}$ of incubation microbial counts reached were $1.22 \mathrm{log}$ ufc $\mathrm{mL}^{-1}$ higher in glucose and $0.6-0.8$ greater in EPS than in the control MM medium without any carbon source added $(p<0.05)$ (Table 1$)$. From that point on, the time course of $B$. fragilis growth was different depending on the carbon source, and microbial population levels remained generally higher in EPS than in glucose at late stages of growth (from $48 \mathrm{~h}$ of incubation) (Table 1 ). Thus, microbial counts in glucose continuously decreased from $24 \mathrm{~h}$ to the end of incubation $(p<0.05)$. In contrast, levels of $B$. fragilis with both EPS remained largely unchanged from 24 to $72 \mathrm{~h}$. From that time population levels decreased with both polymers, this decrease being much more pronounced with EPS R1 than with EPS E44.

The production of fermentation end-products from the catabolism of carbohydrates by $B$. fragilis was notably higher in glucose than in EPS and also in medium with the different EPS than in the medium without carbohydrates added all along the incubation period considered in the study $(p<0.05)$ (Table 1$)$. Glucose was depleted in the culture medium after $24 \mathrm{~h}$ (data not shown) and metabolite levels remained without noticeable variations until $144 \mathrm{~h}$ of incubation. In contrast, total metabolites produced in medium with both EPS increased continuously until the end of fermentation, reaching clearly higher levels at the end of the incubation period in the culture with EPS E44 than in EPS R1 $(p<0.05)$. We analysed more in depth the evolution pattern of the different organic acids and SCFA formed during incubation (Table 1). The acetic to propionic acids ratio displayed higher values in glucose than in both EPS $(p<0.05)$ whereas, conversely, the propionic to succinic as well as the acetic to lactic acid ratios showed a trend $(p<0.1)$ to display higher values in cultures with EPS than with glucose. Regarding levels of the different microbial metabolites in cultures with EPS, the acetic, propionic and succinic acids increased until $24 \mathrm{~h}$, decreased from 24 to $48-72 \mathrm{~h}$, and increased again until the end of incubation. On the opposite, the concentration of lactic acid reached the maximum value at $48 \mathrm{~h}$ and then experienced a continuous decrease until the end of incubation $(p<0.05)$. As a consequence, at $48 \mathrm{~h}$ the acetic to lactic acid ratio reached its minimum value whereas the acetic to propionic ratio attained its maximum in cultures with EPS E44.

Since the only carbon source available in our experimental conditions were either glucose or EPS, we examined the evolution of the EPS fractions over time, looking 
Table 1 Growth and metabolic parameters of B. fragilis grown in glucose, EPS or without carbon source

\begin{tabular}{|c|c|c|c|c|c|c|c|c|c|c|c|}
\hline Carbon source & Time (h) & Acetic acid & Propionic acid & Lactic acid & Succinic acid & Formic acid & Total metabolites* & Acetic/Propionic & Propionic/Succinic & Acetic/Lactic & Bacterial counts $^{+}$ \\
\hline \multirow[t]{4}{*}{ Glucose } & 24 & $13.00 \pm 0.41^{\mathrm{D}}$ & $4.35 \pm 0.63^{C}$ & $14.81 \pm 0.27^{B}$ & $3.46 \pm 0.19^{\mathrm{a} B}$ & $7.33 \pm 0.15^{\mathrm{D}}$ & $42.95 \pm 1.40^{\mathrm{a} C}$ & $3.02 \pm 0.35^{c}$ & $1.25 \pm 0.12^{\mathrm{A}}$ & $0.88 \pm 0.01^{\mathrm{A}}$ & $9.58 \pm 0.07^{d C}$ \\
\hline & 48 & $13.28 \pm 0.74^{C}$ & $4.69 \pm 0.21^{C}$ & $17.11 \pm 1.26^{B}$ & $3.75 \pm 0.27^{\mathrm{a} B}$ & $6.86 \pm 0.38^{\mathrm{D}}$ & $45.73 \pm 1.22^{\mathrm{ab} D}$ & $2.83 \pm 0.08^{\mathrm{D}}$ & $1.25 \pm 0.13^{\mathrm{A}}$ & $0.78 \pm 0.07^{\mathrm{A}}$ & $8.47 \pm 0.16^{C B}$ \\
\hline & 72 & $13.80 \pm 0.53^{C}$ & $5.04 \pm 0.47^{D}$ & $16.15 \pm 1.01^{B}$ & $4.37 \pm 0.18^{b c}$ & $7.59 \pm 0.12^{c}$ & $47.11 \pm 0.41^{b c}$ & $2.75 \pm 0.18^{\mathrm{D}}$ & $1.15 \pm 0.09^{A}$ & $0.86 \pm 0.09$ & $7.76 \pm 0.06^{\mathrm{b} \mathrm{A}}$ \\
\hline & 144 & $13.66 \pm 0.26^{\mathrm{D}}$ & $4.96 \pm 0.32^{\mathrm{D}}$ & $15.27 \pm 0.87^{B}$ & $4.56 \pm 0.52^{\mathrm{b} \mathrm{B}}$ & $7.26 \pm 0.45^{c}$ & $46.02 \pm 1.96^{\mathrm{ab} D}$ & $2.76 \pm 0.15^{c}$ & $1.09 \pm 0.06^{\mathrm{A}}$ & $0.90 \pm 0.05^{\mathrm{A}}$ & $7.46 \pm 0.17^{\mathrm{a} B}$ \\
\hline \multirow[t]{4}{*}{ EPS E44 } & 24 & $1.24 \pm 0.06^{\mathrm{b} B}$ & $0.78 \pm 0.08^{\mathrm{b} A, \mathrm{~B}}$ & $0.45 \pm 0.07^{\mathrm{b} A}$ & $0.12 \pm 0.02^{\mathrm{A}}$ & $0.43 \pm 0.04^{\mathrm{a} B}$ & $3.01 \pm 0.11^{\mathrm{a} B}$ & $1.60 \pm 0.10^{\mathrm{a} B}$ & $6.78 \pm 0.27^{D}$ & $2.83 \pm 0.53^{\mathrm{a} \mathrm{A}}$ & $9.17 \pm 0.12^{C B}$ \\
\hline & 48 & $0.93 \pm 0.08^{\mathrm{a} A, B}$ & $0.39 \pm 0.08^{\mathrm{a} \mathrm{A}}$ & $0.95 \pm 0.05^{\mathrm{CA}}$ & - & $1.27 \pm 0.24^{b c}$ & $3.56 \pm 0.22^{b c}$ & $2.44 \pm 0.30^{b} c$ & ND & $0.98 \pm 0.12^{\mathrm{a} A}$ & $8.91 \pm 0.02^{b c}$ \\
\hline & 72 & $1.89 \pm 0.10^{C B}$ & $0.95 \pm 0.13^{\mathrm{b} B}$ & $0.77 \pm 0.08^{\mathrm{CA}}$ & - & $1.67 \pm 0.46^{\mathrm{b} \mathrm{B}}$ & $5.31 \pm 0.34^{C B}$ & $2.01 \pm 0.19^{\mathrm{ab} C}$ & ND & $2.48 \pm 0.38^{\mathrm{a}}$ & $9.05 \pm 0.05^{b c} B$ \\
\hline & 144 & $5.53 \pm 0.08^{d} C$ & $2.75 \pm 0.13^{c C}$ & $0.26 \pm 0.15^{\mathrm{a} \mathrm{A}}$ & $0.13 \pm 0.05^{\mathrm{A}}$ & $1.40 \pm 0.31^{\mathrm{b} \mathrm{B}}$ & $10.07 \pm 0.39^{d} C$ & $2.01 \pm 0.12^{\mathrm{ab} \mathrm{B}}$ & $25.22 \pm 11.55^{B}$ & $26.24 \pm 12.29^{\mathrm{b} A}$ & $8.68 \pm 0.14^{\mathrm{a} C}$ \\
\hline \multirow[t]{4}{*}{ EPS R1 } & 24 & $1.70 \pm 0.11^{\mathrm{a} C}$ & $1.37 \pm 0.12^{\mathrm{b} B}$ & $0.16 \pm 0.10^{\mathrm{b} \mathrm{A}}$ & $0.26 \pm 0.05^{\mathrm{b} \mathrm{A}}$ & $0.73 \pm 0.25^{\mathrm{a} C}$ & $4.22 \pm 0.09^{\mathrm{a} B}$ & $1.25 \pm 0.03^{\mathrm{a} B}$ & $5.40 \pm 0.54^{b c}$ & $14.03 \pm 7.32^{\mathrm{a} B}$ & $9.01 \pm 0.09^{b B}$ \\
\hline & 48 & $1.40 \pm 0.22^{\mathrm{a} B}$ & $0.82 \pm 0.12^{\mathrm{a} B}$ & $0.49 \pm 0.09^{c A}$ & $0.15 \pm 0.01^{\mathrm{a} \mathrm{A}}$ & $1.92 \pm 0.12^{C B}$ & $4.81 \pm 0.37^{b} \mathrm{~B}$ & $1.70 \pm 0.08^{\mathrm{b} B}$ & $5.64 \pm 0.55^{\mathrm{b} B}$ & $2.86 \pm 0.12^{\mathrm{a} B}$ & $9.17 \pm 0.09^{b} \mathrm{D}$ \\
\hline & 72 & $2.08 \pm 0.10^{\mathrm{b} B}$ & $1.44 \pm 0.16^{b} c$ & $0.12 \pm 0.09^{\mathrm{b} A}$ & $0.26 \pm 0.00^{\mathrm{b} B}$ & $1.35 \pm 0.18^{\mathrm{b} \mathrm{B}}$ & $5.25 \pm 0.09^{b}$ B & $1.45 \pm 0.11^{\mathrm{a} B}$ & $5.62 \pm 0.62^{b}$ B & $25.26 \pm 19.65^{a}$ & $9.15 \pm 0.13^{b ~ B}$ \\
\hline & 144 & $3.13 \pm 0.18^{\mathrm{CB}}$ & $1.70 \pm 0.09^{C B}$ & $0.02 \pm 0.01^{\mathrm{a} \mathrm{A}}$ & $0.53 \pm 0.02^{\mathrm{CA}}$ & $1.27 \pm 0.26^{\mathrm{b} \mathrm{B}}$ & $6.65 \pm 0.34^{C B}$ & $1.85 \pm 0.20^{b} \mathrm{~B}$ & $3.21 \pm 0.29^{\mathrm{a} A}$ & $>100^{\mathrm{b} B}$ & $6.73 \pm 0.21^{\mathrm{a} \mathrm{A}}$ \\
\hline \multirow[t]{4}{*}{ WCS } & 24 & $0.21 \pm 0.05^{\mathrm{a} A}$ & $0.26 \pm 0.03^{\mathrm{a} A}$ & - & $0.11 \pm 0.07^{\mathrm{a} \mathrm{A}}$ & $0.09 \pm 0.03^{\mathrm{a} \mathrm{A}}$ & $0.72 \pm 0.12^{\mathrm{a} A}$ & $0.79 \pm 0.12^{\mathrm{A}}$ & $2.33 \pm 0.43^{B}$ & ND & $8.36 \pm 0.15^{\mathrm{CA}}$ \\
\hline & 48 & $0.27 \pm 0.05^{\mathrm{a} \mathrm{A}}$ & $0.34 \pm 0.03^{\mathrm{b} \mathrm{A}}$ & - & $0.21 \pm 0.01^{\mathrm{b} \mathrm{A}}$ & $0.17 \pm 0.03^{\mathrm{b} A}$ & $1.01 \pm 0.05^{\mathrm{a} \mathrm{A}}$ & $0.79 \pm 0.10^{A}$ & $1.66 \pm 0.21^{\mathrm{A}}$ & ND & $8.10 \pm 0.06^{\mathrm{CA}}$ \\
\hline & 72 & $0.35 \pm 0.06^{\mathrm{a} A}$ & $0.36 \pm 0.00^{\mathrm{b} \mathrm{A}}$ & - & $0.20 \pm 0.03^{\mathrm{b} \mathrm{A}}$ & $0.17 \pm 0.04^{\mathrm{b} A}$ & $1.08 \pm 0.02^{\mathrm{a} A}$ & $0.99 \pm 0.16^{\mathrm{A}}$ & $1.83 \pm 0.29^{\mathrm{A}}$ & ND & $7.84 \pm 0.11^{\mathrm{b} \mathrm{A}}$ \\
\hline & 144 & $0.66 \pm 0.22^{\mathrm{b} \mathrm{A}}$ & $0.51 \pm 0.05^{\mathrm{CA}}$ & - & $0.23 \pm 0.05^{\mathrm{b} \mathrm{A}}$ & $0.26 \pm 0.03^{\mathrm{cA}}$ & $1.68 \pm 0.35^{\mathrm{b} \mathrm{A}}$ & $1.28 \pm 0.30^{\mathrm{A}}$ & $2.30 \pm 0.36^{A}$ & ND & $6.61 \pm 0.19^{\mathrm{a} A}$ \\
\hline
\end{tabular}

Metabolite concentrations are expressed in $\mathrm{mM}$, bacterial counts are expressed in ufc $\mathrm{mL}^{-1}$ Columns for the same carbon source with different lower case letter superscripts indicate significant differences along time $(p>0.05)$. Different capital letters superscripts indicate significant differences among carbon sources at the same sampling point $(p<0.05)$. ${ }^{*}$ Sum of acetic, propionic, lactic, succinic, formic and pyruvic acids. ${ }^{+}$Counts at $0 \mathrm{~h}$ were $7.02 \pm 0.08 \log$ ufc $\mathrm{mL}^{-1}$. WCS without carbon source. -, under the detection limit, ND Not determined 
for detectable variations in the amount and/or physicochemical characteristics of the polymer peaks that could demonstrate their consumption. Peaks of EPS E44, purified from the cellular biomass of Bif. longum E44, were according to that previously reported [22] and consisted in two polymers, one of $5 \times 10^{6} \mathrm{Da}$ (E44 P1) representing $18.8 \%$ of the total mass, and a second one of $9 \times 10^{3} \mathrm{Da}$ (E44 P2), representing $81.2 \%$ (Additional file 2: Figure S2). We ruled out any change in the molar mass distribution of the EPS E44 fraction along incubation in uninoculated MM (data not shown). Interestingly, the incubation of EPS with B. fragilis in the MM resulted in a significant decrease in the amount of the higher molar mass peak (E44 P1) of E44 from 48 h to $144 \mathrm{~h}$ of incubation $(p<0.05)$ (Fig. 1a and b) and of the smaller molar mass peak (E44 P2) between 48 and $72 \mathrm{~h}$ of incubation, remaining stable from 72 to $144 \mathrm{~h}(p<0.05)$ (Fig. 1a and b). Molar mass distribution of EPS R1 was also in accordance with that previously reported by us [24], and consisted of three peaks; one of $1 \times 10^{6} \mathrm{Da}$ (R1 P1), another one of $2.35 \times 10^{4} \mathrm{Da}$ (R2 P2) and a small one of $4 \times 10^{3} \mathrm{Da}$ (R3 P3). This last peak overlapped in our analysis with an UV absorption peak and was then excluded from our study (Additional file 2: Figure S2). The total amount of the higher molar mass R1 polymer (R1 P1) did not show a decrease at the end of incubation, independently on the presence of $B$. fragilis (Fig. 1d). The amount of the medium size polymer (R1 P2) suffered an apparent depletion along incubation, which reached statistical significance at late incubation times $(48,72$ and $144 \mathrm{~h}, p<0.05)$; however, the final amount of this polymer in the MM without $B$. fragilis was not significantly different $(p>0.05)$ from the values obtained in the culture with bacteria added (Fig. 1c and d) (statistical data not shown).

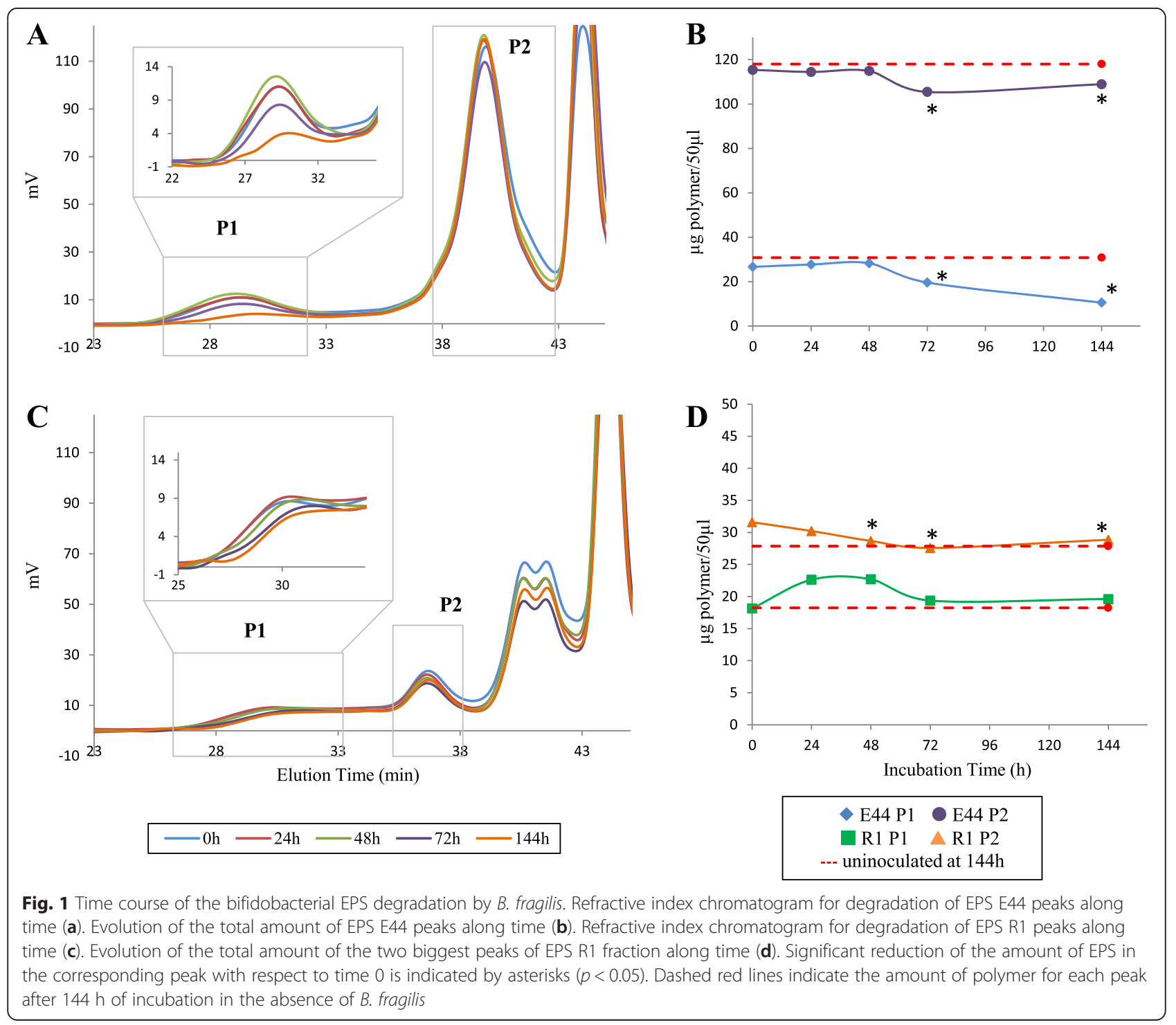




\section{Discussion}

The use of bacterial EPS by Bacteroides thetaiotaomicron has been described before with HoPS produced by some lactic acid bacteria and Streptococcus spp. [6, 16, 25]. Although the use of HePS from bifidobacteria had not been definitely demonstrated yet, we have previously identified changes in the metabolism of $B$. fragilis in the presence of these polymers in an undefined medium [18]. Results from the current study demonstrated an effective growth of this specie in the presence of HePS. As compared to glucose, Bacteroides population levels attained with our EPS at early stages of growth were lower, but probably the slow utilization of these polymers and hence their availability as carbon source along incubation contributed to maintain the microbial levels at late states of growth, this phenomenon being more pronounced with EPS E44 than with EPS R1. Besides, a differential metabolic activity of $B$. fragilis in the presence of EPS as compared to glucose was evidenced. B. fragilis remained metabolically active in cultures with EPS for a longer period of time, with the highest activity corresponding to the cultures with the polymer E44. Variations obtained in metabolic profiles of Bacteroides cultures as depending on the carbon sources were similar to those indicated previously by us using an undefined medium [18]. Additionally, higher propionic to succinic acid ratios with complex carbon sources relative to glucose, similar to that found in the current study, have been previously reported [26].

In the present study we observed a clear shift in the metabolite production by $B$. fragilis during the time course of fermentation in the presence of EPS with respect to glucose, which was more pronounced with the polymer E44 than with R1. Coinciding with the depletion in the amount of EPS polymers from $48 \mathrm{~h}$ of incubation, a gradual increase in the concentrations of acetic, propionic and succinic acids and a decrease in the levels of lactic acid occurred until the end of incubation. It has been previously suggested that, in Bacteroides, the production of propionate through the succinate/propionate pathway could be a cell response to optimize cell energy production while keeping the intracellular redox balance $[18,19]$. Although $B$. fragilis has the capacity to metabolize moderate amounts of lactic acid [27] as well as amino acids [28], our work was performed in a minimal medium, so that no amino acid sources were available and the scarce consumption of lactic acid that may occur does not explain the growth and metabolic activity of the bacterium in such conditions.

The results from SEC-MALS chromatography indicated that B. fragilis was able to use the EPS E44 produced by Bif. longum as a fermentable substrate. The chemical structure of EPS E44 has not been elucidated yet but it is known that EPS E44 contains glucose and galactose in its composition [22]. It is then possible that the saccharolytic enzymatic machinery of $B$. fragilis could include enzymes able to participate in the degradation of this complex substrate. On the other hand, changes in growth and metabolic patterns occurring at late stages of growth in EPS E44 could be related with the cessation of consumption of the smaller peak beyond $72 \mathrm{~h}$ of incubation, and hence with a scarcity of carbon source available from this time. Our results are not conclusive about the possible degradation of the EPS R1 fraction by $B$. fragilis. Even though there were no significant changes attributable to the activity of $B$. fragilis in the EPS peaks of high and medium molar mass, we could not rule out changes in the amount of the smallest polymer, not considered in our study because the overlapping with a protein peak. Variations in this small polymer may provide a possible explanation for the increased metabolic activity evidenced in cultures of $B$. fragilis until $72 \mathrm{~h}$ of incubation in the presence of the EPS R1 fraction as compared to the control in MM without carbon sources added. The EPS R1 fraction is formed by glucose, galactose and rhamnose [24] and only the chemical structure of the high molar mass peak has been determined to date, which is composed by $50 \%$ rhamnose [29]. Although we know that the presence of both EPS stimulates the production of $\alpha$ glucosidase by $B$. fragilis, the high molar mass polymer of the EPS R1 fraction lacks the $\alpha$-linkages targeted by this enzymatic activity [29]. This together with the inability of $B$. fragilis to ferment rhamnose [28], could pose difficulties for the use as fermentable substrate of the EPS R1 by this microorganism.

The ability of $B$. fragilis to use bifidobacterial EPS may provide this microorganism with a long-term available complex carbon source, thus enhancing its survival and conferring it a selective advantage in environments where nutrients are scarce, such as the case of the human large intestine. Bacteroides plays an important role in the utilization of indigestible dietary compounds and complex polymers secreted by other microorganisms [6]. Some EPS and capsular polysaccharides are involved in adhesion to eukaryotic cells, biofilm formation and protection of several species against the gastrointestinal stressing factors [14]. In this way the ability of Bacteroides to degrade these polymers may confer this microorganism a role in the regulation of microbial relationships in the gut ecosystem. Fermentation of EPS in faecal cultures lead to an increase in propionic acid production [17], most likely due to the metabolic activity of members from the genus Bacteroides.

\section{Conclusions}

The present work is the first report demonstrating that B. fragilis is able to use some EPS produced by a bifidobacteria as substrate for growth, which resulted in a partial polymer consumption and the concomitant release 
of metabolic end products from its fermentation. By extrapolating these findings to the human gut, it may be hypothesized that the feeding relationship between microbial EPS and Bacteroides could have an impact in the SCFA production balance in the gut, which is ultimately related with the human health.

\section{Additional files}

Additional file 1: Figure S1. Thin layer chromatography of the digestion of glycogen and EPS E44 and R1 fractions with enzymes a-amylase, pullulanase, and with a mixture of a-amylase and pullulanase. (PPTX $522 \mathrm{~kb}$ )

Additional file 2: Figure S2. Size exclusion chromatography (SEC-MALS) analysis of the EPS E44 (A) and EPS R1 (B) fractions purified from the cell biomass of Bifidobacterium longum E44 and Bifidobacterium animalis subsp. lactis R1, respectively. Refractive index detector (blue line) for detection and quantification of EPS peaks, PDA detector (green line) set at $280 \mathrm{~nm}$ to identify the presence of proteins, and the multiangle laser light scattering (MALS) for molar mass distribution of the EPS fractions (red line). (PPTX $758 \mathrm{~kb}$ )

\section{Abbreviations}

EPS, Exopolysaccharide; GAMc, Gifu Anaerobic Medium with cysteine; HePS Heteroexopolysaccharides; HoPS, Homopolysaccharides; MM, Minimal medium; SCFA, Short Chain Fatty Acids; SEC-MALLS, Size-Exclusion chromatographyMultiangle Laser Light Scattering

\section{Acknowledgments}

David Ríos-Covián was the recipient of a predoctoral FPI fellowship granted by MINECO. We would like to thank Lidia Alaez for her excellent technical assistance.

\section{Funding}

This work was financed by projects AGL2010-16525 and AGL2013-43770-R from Plan Nacional/Plan Estatal de I + D + I (Spanish Ministry of Economy and Competitiveness, MINECO) and the Grant GRUPIN14-043 from "Plan Regional de Investigación del Principado de Asturias". Both, national and regional grants received cofounding from European Union FEDER funds.

\section{Availability of data and materials}

All the data supporting these paper findings is contained within the article and in the supplementary files.

\section{Authors' contribution}

DRC participated in the design of the experiment and carried out the bacterial cultures, data analysis and interpretation of results, and drafted the manuscript. IC carried out the HPLC analysis. JRA performed the GC analysis. PRM participated in the analysis and interpretation of MALLS data. MG participated in the study design and data interpretation. CGR participated in the study design and data interpretation. All authors revised and approved the final manuscript.

\section{Competing interests}

The authors declare that they have no competing interests.

\section{Consent to publish}

Not applicable.

\section{Ethics and consent to participate}

Not applicable.

\section{Author details}

${ }^{1}$ Probiotics and Prebiotics Group, Department of Microbiology and Biochemistry of Dairy Products, Instituto de Productos Lácteos de Asturias, Consejo Superior de Investigaciones Científicas (IPLA-CSIC), Villaviciosa, Asturias, Spain. ${ }^{2}$ Scientific and Technical Facilities, Instituto de Productos
Lácteos de Asturias, Consejo Superior de Investigaciones Científicas (IPLA-CSIC), Villaviciosa, Asturias, Spain.

Received: 27 January 2016 Accepted: 12 July 2016

Published online: 15 July 2016

\section{References}

1. Flint HJ, Duncan SH, Scott KP, Louis P. Links between diet, gut microbiota composition and gut metabolism. Proc Nutr Soc. 2015:74:13-22.

2. Turnbaugh PJ, Ridaura VK, Faith JJ, Rey FE, Knight R, Gordon Jl. The effect of diet on the human gut microbiome: a metagenomic analysis in humanized gnotobiotic mice. Sci Transl Med. 2009;1:6ra14.

3. Rigottier-Gois L, Bourhis AG, Gramet G, Rochet V, Dore J. Fluorescent hybridisation combined with flow cytometry and hybridisation of total RNA to analyse the composition of microbial communities in human faeces using 165 rRNA probes. FEMS Microbiol Ecol. 2003;43:237-45.

4. Lozupone CA, Hamady M, Cantarel BL, Coutinho PM, Henrissat B, Gordon Jl, Knight R. The convergence of carbohydrate active gene repertoires in human gut microbes. Proc Natl Acad Sci U S A. 2008;105:15076-81.

5. Xu J, Mahowald MA, Ley RE, Lozupone CA, Hamady M, Martens EC, et al. Evolution of symbiotic bacteria in the distal human intestine. PLoS Biol. 2007:5:e156

6. Sonnenburg ED, Zheng H, Joglekar P, Higginbottom SK, Firbank SJ, Bolam DN, Sonnenburg JL. Specificity of polysaccharide use in intestinal bacteroides species determines diet-induced microbiota alterations. Cell. 2010;141:1241-52

7. Wexler HM. Bacteroides: the good, the bad, and the nitty-gritty. Clin Microbiol Rev. 2007;20:593-621.

8. Mahowald MA, Rey FE, Seedorf H, Turnbaugh PJ, Fulton RS, Wollam A, et al. Characterizing a model human gut microbiota composed of members of its two dominant bacterial phyla. Proc Natl Acad Sci U S A. 2009;106:5859-64.

9. Namavar F, Theunissen EB, Verweij-Van Vught AM, Peerbooms PG, Bal M, Hoitsma HF, MacLaren DM. Epidemiology of the Bacteroides fragilis group in the colonic flora in 10 patients with colonic cancer. J Med Microbiol. 1989:29:171-6.

10. Mazmanian SK, Liu CH, Tzianabos AO, Kasper DL. An immunomodulatory molecule of symbiotic bacteria directs maturation of the host immune system. Cell. 2005;122:107-18.

11. Kuwahara T, Yamashita A, Hirakawa H, Nakayama H, Toh H, Okada N, Kuhara S, Hattori M, Hayashi T, Ohnishi Y. Genomic analysis of Bacteroides fragilis reveals extensive DNA inversions regulating cell surface adaptation. Proc Natl Acad Sci U S A. 2004;101:14919-24.

12. Ruas-Madiedo P, Moreno JA, Salazar N, Delgado S, Mayo B, Margolles A, de los Reyes-Gavilan CG. Screening of exopolysaccharide-producing Lactobacillus and Bifidobacterium strains isolated from the human intestinal microbiota. Appl Environ Microbiol. 2007;73:4385-8.

13. Ruas-Madiedo P, Gueimonde M, Arigoni F, de los Reyes-Gavilan CG, Margolles A. Bile affects the synthesis of exopolysaccharides by Bifidobacterium animalis. Appl Environ Microbiol. 2009;75:1204-7.

14. Hidalgo-Cantabrana C, Sánchez B, Milani C, Ventura M, Margolles A, Ruas-Madiedo P. Genomic overview and biological functions of exopolysaccharide biosynthesis in Bifidobacterium spp. Appl Environ Microbiol. 2014:80:9-18.

15. Salazar N, Gueimonde M, Hernandez-Barranco AM, Ruas-Madiedo P, de los Reyes-Gavilan CG. Exopolysaccharides produced by intestinal Bifidobacterium strains act as fermentable substrates for human intestinal bacteria. Appl Environ Microbiol. 2008:74:4737-45.

16. Grosu-Tudor SS, Zamfir M, Meulen RVD, Falony G, Vuyst LC. Prebiotic potential of some exopolysaccharides produced by lactic acid bacteria. Rom Biotechnol Lett. 2013;18:8666-76.

17. Salazar N, Ruas-Madiedo P, Kolida S, Collins M, Rastall R, Gibson G, de los Reyes-Gavilan CG. Exopolysaccharides produced by Bifidobacterium longum IPLA E44 and Bifidobacterium animalis subsp. lactis IPLA R1 modify the composition and metabolic activity of human faecal microbiota in $\mathrm{pH}$ controlled batch cultures. Int J Food Microbiol. 2009;135:260-7.

18. Rios-Covian D, Sanchez B, Salazar N, Martinez N, Redruello B, Gueimonde M, de los Reyes-Gavilan CG. Different metabolic features of Bacteroides fragilis growing in the presence of glucose and exopolysaccharides of bifidobacteria. Front Microbiol. 2015;6:825.

19. Sperry JF, Appleman MD, Wilkins TD. Requirement of heme for growth of Bacteroides fragilis. Appl Environ Microbiol. 1977;34:386-90. 
20. Ruas-Madiedo P, Gueimonde M, Margolles A, de los Reyes-Gavilan CG Salminen S. Exopolysaccharides produced by probiotic strains modify the adhesion of probiotics and enteropathogens to human intestinal mucus. J Food Prot. 2006:69:2011-5.

21. Delgado S, Ruas-Madiedo P, Suarez A, Mayo B. Interindividual differences in microbial counts and biochemical-associated variables in the feces of healthy Spanish adults. Dig Dis Sci. 2006;51:737-43.

22. Koropatkin NM, Smith TJ. SusG: A unique cell-membrane-associated a-amylase from a prominent human gut symbiont targets complex starch molecules. Structure. 2010;18:200-15. doi: http://dx.doi.org/10.1016/j.str.2009.12.010.

23. Salazar N, Prieto A, Leal JA, Mayo B, Bada-Gancedo JC, de los Reyes-Gavilan CG, Ruas-Madiedo P. Production of exopolysaccharides by Lactobacillus and Bifidobacterium strains of human origin, and metabolic activity of the producing bacteria in milk. J Dairy Sci. 2009;92:4158-68.

24. Ruas-Madiedo P, Medrano M, Salazar N, de los Reyes-Gavilan CG, Perez PF, Abraham AG. Exopolysaccharides produced by Lactobacillus and Bifidobacterium strains abrogate in vitro the cytotoxic effect of bacterial toxins on eukaryotic cells. J Appl Microbiol. 2010;109:2079-86.

25. van Bueren AL, Saraf A, Martens EC, Dijkhuizena L. Differential metabolism of exopolysaccharides from probiotic lactobacilli by the human gut symbiont Bacteroides thetaiotaomicron. Appl Environ Microbiol. 2015;81:3973-83.

26. Adamberg S, Tomson K, Vija H, Puurand M, Kabanova N, Visnapuu T, Jõgi $E$, Alamäe T, Adamberg K. Degradation of fructans and production of propionic acid by Bacteroides thetaiotaomicron are enhanced by shortage of amino acids. Front Nutr. 2014;1:21.

27. Rios-Covian D, Arboleya S, Hernandez-Barranco AM, Alvarez-Buylla JR, RuasMadiedo P, Gueimonde M, de los Reyes-Gavilan CG. Interactions between Bifidobacterium and Bacteroides species in cofermentations are affected by carbon sources, including exopolysaccharides produced by bifidobacteria. Appl Environ Microbiol. 2013;79:7518-24.

28. Wexler HM. The genus Bacteroides. In: The Prokaryotes: Other Major Lineages of Bacteria and The Archaea. 2013. p. 459-84.

29. Leivers S, Hidalgo-Cantabrana C, Robinson G, Margolles A, Ruas-Madiedo P, Laws AP. Structure of the high molecular weight exopolysaccharide produced by Bifidobacterium animalis subsp. lactis IPLA-R1 and sequence analysis of its putative eps cluster. Carbohydr Res. 2011;346:2710-7.

\section{Submit your next manuscript to BioMed Central and we will help you at every step:}

- We accept pre-submission inquiries

- Our selector tool helps you to find the most relevant journal

- We provide round the clock customer support

- Convenient online submission

- Thorough peer review

- Inclusion in PubMed and all major indexing services

- Maximum visibility for your research

Submit your manuscript at www.biomedcentral.com/submit

CBiomed Central 